\title{
Protective effects of DL-3-n-butylphthalide in the lipopolysaccharide-induced mouse model of Parkinson's disease
}

\author{
MU-JUN JIANG ${ }^{1 *}$, YU-HUA CHEN ${ }^{1 *}$, LI LI $^{1 *}$, LI XU $^{1}$, HAO LIU $^{2}$, XIAO-LONG QU ${ }^{3}$, \\ JUAN-JUAN XU ${ }^{1}$, BO-BO GE ${ }^{1}$ and HONG-DANG QU ${ }^{1}$ \\ ${ }^{1}$ Department of Neurology, The First Affiliated Hospital of Bengbu Medical College; ${ }^{2}$ Faculty of Pharmacy, \\ Bengbu Medical College; ${ }^{3}$ Department of Anesthesiology, The Second Affiliated Hospital of \\ Bengbu Medical College, Bengbu, Anhui 233040, P.R. China
}

Received November 2, 2016; Accepted June 22, 2017

DOI: $10.3892 / \mathrm{mmr} .2017 .7352$

\begin{abstract}
DL-3-n-butylphthalide (NBP) is extracted from rapeseed and exhibits multiple neuroprotective effects, exerted by inhibiting the inflammatory process, including reducing oxidative stress, improving mitochondrial function and reducing neuronal apoptosis. The present study aimed to investigate the neuroprotective effects of NBP in a lipopolysaccharide (LPS)-induced mouse model of Parkinson's disease (PD). The behavior of mice was assessed using the rotarod test and open-field test, the amount of tyrosine hydroxylase in the substantia nigra pars compacta was evaluated by immunohistochemistry, and the levels of phosphorylated c-Jun N-terminal kinase (JNK), mitogen-activated protein kinase 14 (p38) and extracellular signal-regulated kinase 1 were determined by western blotting. It was demonstrated that the LPS-induced behavioral deficits were significantly improved. LPS-induced dopaminergic neurodegeneration was relieved following treatment with NBP, as determined from tyrosine hydroxylase-positive cells. Phosphorylation of JNK and p38 was significantly inhibited following treatment with NBP. Therefore in the present study, a role for NBP has been established in the treatment of a PD murine model, laying the experimental basis for the treatment of PD with this agent.
\end{abstract}

Correspondence to: Professor Hong-Dang Qu, Department of Neurology, The First Affiliated Hospital of Bengbu Medical College, 287 Changhuai Road, Bengbu, Anhui 233040, P.R. China

E-mail: qhd820@sohu.com

*Contributed equally

Abbreviations: NBP,DL-3-n-butylphthalide; PD,Parkinson's disease; $\mathrm{TH}$, tyrosine hydroxylase; SNpc, substantia nigra pars compacta; MAPK, mitogen-activated protein kinase; LPS, lipopolysaccharide; MPTP, methyl-4-phenyl-1,2,3,6-tetrahydropyridine; 6-OHDA, 6-hydroxydopamine

Key words: DL-3-n-butylphthalide, lipopolysaccharide, mouse model, Parkinson's disease, tyrosine hydroxylase

\section{Introduction}

Parkinson's disease (PD) is a common neurodegenerative disorder, which is caused by degeneration of dopaminergic neurons in the substantia nigra pars compacta (SNpc) (1). Despite a number of studies, the accurate pathogenesis of PD remains unclear and increasing evidence has demonstrated that activation of the microglia, with the attendant oxidative stress and neuroinflammation, may be crucial events in the pathogenesis of PD $(2,3)$. Additionally, it has been demonstrated that the mitogen-activated protein kinase (MAPK) pathway serves an important role in the pathogenesis of PD (4). Modulating the MAPK pathway represents a promising approach for the prevention and treatment of $\mathrm{PD}$.

In the past few years, several neurotoxic molecules, including 6-hydroxydopamine (6-OHDA) and methyl-4phenyl-1,2,3,6-tetrahydropyridine (MPTP), have been utilized to establish PD models, by causing an inflammatory response; however, it is difficult to confirm whether neuroinflammation is the consequence or cause in injured dopaminergic neurons (5). However, a PD model induced by lipopolysaccharide (LPS), as an effective glial cell activator, has demonstrated a gradual and selective reduction in dopaminergic neurons in the SNpc (6). Several clinical and basic studies have indicated that LPS-induced PD models demonstrate the basic characteristics of PD pathology, including anti-inflammatory actions (similar to nonsteroidal antiinflammatory drugs, cyclo-oxgenase-2 inhibitors, inducible nitric oxide synthase inhibitors) and anti-oxidative properties, particularly NADPH inhibitors (7-9).

In south China, DL-3n-butylphthalide (NBP) is extracted from rapeseed and was approved for clinical use by the China Food and Drug Administration in 2002 (Beijing, China). NBP has been demonstrated to possess multiple neuroprotective effects exerted by inhibiting the inflammatory process including reducing oxidative stress, improving mitochondrial function and reducing neuronal apoptosis $(10,11)$. NBP also suppresses the generation of peroxynitrite, superoxide and nitric oxide (12). Previously, NBP has been reported to be able to prevent oxidative damage and reduce mitochondrial dysfunction in a 1-methyl-4-phenylpyridinium ${ }^{+}$-induced cellular model of PD (13). 
Therefore, in the present study, the neuroprotective effects of NBP on LPS-induced inflammatory nerve damage in a PD mouse model were examined and the possible mechanisms were investigated.

\section{Materials and methods}

Reagents. Anti-c-Jun N-terminal kinase (JNK), MAPK 14 (p38) and extracellular signal-regulated kinase (ERK), phosphorylated (p)-p38, p-JNK, and p-ERK rabbit antibodies were purchased from Cell Signaling Technology, Inc. (Danvers, MA, USA; cat. nos. 9252, 9212, 4695, 9211, 9251 and 9101, respectively). Anti- $\beta$-actin rabbit polyclonal antibody was purchased from Beyotime Institute of Biotechnology (Haimen, China; cat. no. AA128). Protease inhibitor cocktail and extraction buffer [radioimmunoprecipitation assay (RIPA) lysis buffer; cat. no. P0013B] were purchased from the Beyotime Institute of Biotechnology. Enhanced chemiluminescence (ECL) reagents were purchased from Nanjing KeyGen Biotech Co., Ltd. (Nanjing, China). LPS was obtained from Sigma-Aldrich (Merck KGaA, Darmstadt, Germany) and NBP was obtained from CSPC Pharmaceutical Co., Ltd. (Shijiazhuang, China).

Animals and treatment. Adult male C57BL/6 mice $(n=30)$ weighing 18-20 g were purchased from the Comparative Medicine Center at Yangzhou University [Yangzhou, China; cat. no. $\operatorname{scxk}(\mathrm{Su})$ 2012-004] and housed under pathogen-free conditions with a 12 -h light/dark cycle, $60-70 \%$ relative humidity and temperature of $20-22^{\circ} \mathrm{C}$, and received food and water ad libitum in their cages. All animals were $\sim 10$ weeks of age at the start of administration. Prior to the experiment, the animals were allowed 10 days to adapt to the new environment. All mice were randomly separated into 3 groups ( $n=10 /$ group) as follows: i) The control group, which was treated with saline; ii) the LPS-treatment group, which received a single intraperitoneal injection of LPS at $5 \mathrm{mg} / \mathrm{kg}$ to generate the PD model; and iii) the NBP-treatment group, which received gastric perfusions of NBP $(120 \mathrm{mg} / \mathrm{kg})$ dissolved in soybean oil once a day for 30 days, following injection with LPS (similar to the LPS-treatment group). All experiments commenced following 7 months of treatment. The present study was approved by the ethics committee of Bengbu Medical College (Bengbu, China).

Rotarod test. The rotarod test is widely used to assess motor and coordination abilities, particularly bradykinesia in mice (14). In the present study, a rotarod (3-cm diameter) was utilized at a fixed speed of $20 \mathrm{rpm}$. The retention time for each mouse resting on the revolving rod was recorded. The rotarod test was repeated 3 times for each mouse, with 30-min rest periods in between the tests. The retention time of each mouse was determined and used for comparison.

Open-field test. Following treatment, motor behavior was analyzed in an open-field test. The apparatus consisted of a square $(40 \times 40 \mathrm{~cm})$ with a surrounding wall (height, $30 \mathrm{~cm})$. The square floor was divided into 16 (4x4) sub-squares using transverse and longitudinal segments. Mice were placed in the center of the structure and then the behavior of the mice was recorded for $5 \mathrm{~min}$. When each mouse repeated the test
3 times, their spontaneous activity was analyzed for $30 \mathrm{~min}$. The line crossings, grooming and rearing of each mouse were recorded and analyzed.

Immunohistochemistry. Following treatment, 3 mice were randomly selected from each group for the subsequent study. Mice were deeply anesthetized with $10 \%$ chloral hydrate ( $3 \mathrm{ml} / \mathrm{kg}$; Sigma-Aldrich) at room temperature and perfused through the ventriculus sinister with $100 \mathrm{ml}$ saline, followed by $4 \%$ paraformaldehyde for $20 \mathrm{~min}$. The midbrains were collected and fixed with $4 \%$ paraformaldehyde at $4{ }^{\circ} \mathrm{C}$ for $24 \mathrm{~h}$. Subsequently, the midbrains were embedded in paraffin and cut (35- $\mu$ m-thick) and processed for immunohistochemistry. The sections were blocked with $5 \%$ bovine serum albumin (cat. no. P0007; Beyotime Institute of Biotechnology) dissolved in PBS at room temperature for $1 \mathrm{~h}$.

The sections were incubated with primary monoclonal antibodies at $37^{\circ} \mathrm{C}$ overnight [rabbit anti-tyrosine hydroxylase (TH); 1:1,000; cat. no. 2791; Cell Signaling Technology, Inc.]. Then, the sections were rinsed with PBS, incubated with horseradish peroxidase (HRP)-conjugated secondary antibodies at room temperature for $50 \mathrm{~min}$ (goat anti-rabbit Immunoglobulin G, 1:800 (cat. no. GB23303; Goodbio Technology Co., Ltd., Wuhan, China). Sections were subsequently incubated for $30 \mathrm{~min}$ in peroxidase substrate mixing liquid and then washed and detected with a DAB staining kit (cat. no. K5007; Dako; Agilent Technologies, Santa Clara, CA, USA) at room temperature for 3-10 min, terminating the coloration when brown granules (positive cells) were observed.

Finally, the sections were counterstained with hematoxylin at room temperature for $3 \mathrm{~min}$, dehydrated with deionized water, dried and sealed. The immunopositive cells in the stained sections were analyzed under an optical microscope (Nikon Corporation, Tokyo, Japan). The number of TH-positive cells (magnification, $\mathrm{x} 40$ ) in every sixth section was counted by two researchers blinded to the treatment.

Western blot analysis. RIPA lysis buffer was used to extract proteins from the ventral mesencephalon. Tissues were lyzed in RIPA buffer supplemented with protease inhibitor cocktail. Then, the lysates were centrifuged $(8,000 \mathrm{x} \mathrm{g}$ for $10 \mathrm{~min}$ at $4^{\circ} \mathrm{C}$ ) and the protein concentration in the extracts was determined using the Bradford assay.

Equal amounts $(30 \mu \mathrm{g})$ of protein samples were electrophoresed on $12 \%$ denaturing polyacrylamide gels and then transferred onto nitrocellulose membranes (EMD Millipore, Billerica, MD, USA) using a semi-dry blotting apparatus (Bio-Rad Laboratories, Inc., Hercules, CA, USA) for $2 \mathrm{~h}$ at $4^{\circ} \mathrm{C}$. The membrane was then blocked with $5 \%$ bovine serum albumin (cat. no. P0007; Beyotime Institute of Biotechnology) dissolved in TBS containing Tween [TBST; $10 \mathrm{mM}$ Tris-HCl (pH 7.5), $150 \mathrm{mM} \mathrm{NaCl}$ and $0.1 \%$ Tween-20] at room temperature for $1 \mathrm{~h}$. Following washing with TBST buffer 3 times, membranes were incubated with primary antibodies against JNK, ERK, p38, p-JNK, p-ERK and p-p38 (all 1:1,000) at $4^{\circ} \mathrm{C}$ overnight. Then, the membranes were washed 3 times with TBST for $10 \mathrm{~min}$ and incubated with the HRP-conjugated secondary antibodies (cat. no. GB23303; Goodbio Technology Co., Ltd.) at room temperature for $1 \mathrm{~h}(1: 5,000)$. An anti- $\beta$-actin antibody $(1: 1,000)$ was used as an internal control. 

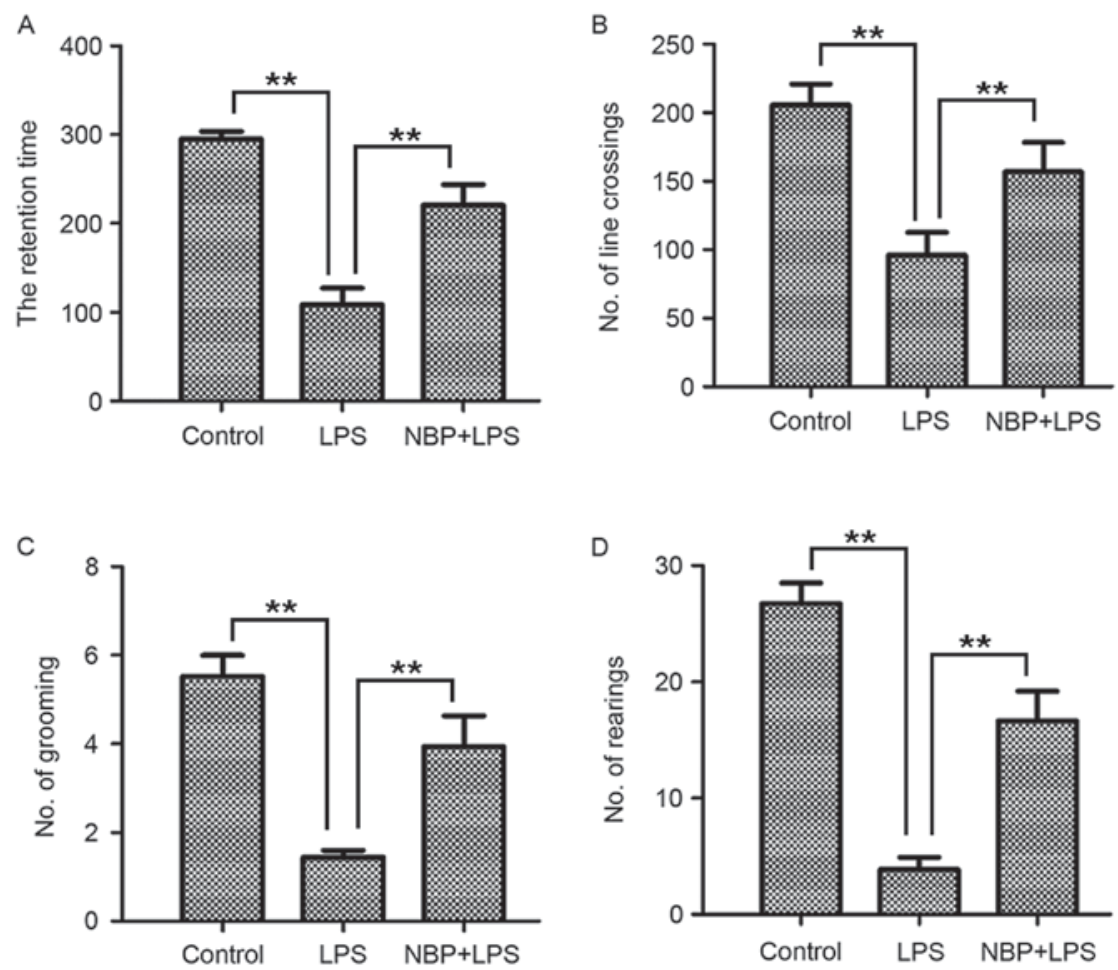

Figure 1. NBP attenuates behavioral impairment in intranasal LPS-induced Parkinson's disease model mice, as assessed by the rotarod test and open field test. (A) Retention times during which each mouse rested on the revolving rod in the control group, LPS-treatment group and NBP-treatment group. Number of (B) line crossing, (C) grooming and (D) rearing actions of each mouse, in the control group, LPS-treatment group and NBP-treatment group. Data are representative of three independent experiments. The values are expressed as the mean \pm standard deviation. ${ }^{* *} \mathrm{P}<0.01$. NBP, DL-3-n-butylphthalide; PD, Parkinson's disease; LPS, lipopolysaccharide.

Band intensities were detected with ECL reagents (cat. no. KGP1123; Nanjing KeyGen Biotech Co., Ltd.) and measured with Quantity one Software version 25.0.0 (Bio-Rad Laboratories, Inc.).

Statistical analysis. All values were expressed as the mean \pm standard deviation. Results were analyzed using one-way analysis of variance followed by Tukey post-hoc tests using SPSS software version 18.0 (SPSS, Inc., Chicago, IL, USA). $\mathrm{P}<0.05$ was considered to indicate a statistically significant difference.

\section{Results}

NBP attenuates behavioral impairment in intraperitoneal $L P S$-induced PD mice. To investigate whether NBP protects mice from LPS-induced neurotoxicity, the behavioral tests were conducted, including the rotarod and the open-field test.

Rotarod test results demonstrated that the retention time of the control group was $298.61 \pm 2.94 \mathrm{sec}$ (Fig. 1A). Compared with the control group, the LPS-treatment group $(103.78 \pm 46.01 \mathrm{sec})$ had a significantly reduced retention time $(\mathrm{P}<0.01)$. However, the NBP-treatment group, who demonstrated marked improvement in retention time (Fig. 1A), had significantly enhanced retention times $(221.83 \pm 53.88 \mathrm{sec})$ compared with mice in the group treated with LPS $(\mathrm{P}<0.01)$.

As presented in Fig. 1B-D, the open field test, which assessed the degree of locomotory capacity demonstrated that the number of line crossing, rearing and grooming actions in the control group was $205.56 \pm 26.82,27.06 \pm 3.59$ and $5.50 \pm 0.84$, respectively. In the LPS-treatment group, the line crossing (96.00 \pm 28.65$)$, rearing $(3.83 \pm 1.87)$ and grooming $(1.44 \pm 0.27)$ activities were decreased significantly (all $\mathrm{P}<0.01$; Fig. 1B-D). In the NBP treatment group, the line crossing (156.67 \pm 37.23$)$, rearing $(16.78 \pm 4.64)$ and grooming $(3.94 \pm 1.22)$ behaviors were significantly increased compared with the LPS-treatment group (all $\mathrm{P}<0.01$; Fig. 1B-D). Therefore, the mice treated with NBP demonstrated a significant improvement in behavioral performance in the open field test compared with the LPS-treatment group.

These results demonstrated that NBP could protect mice from LPS-induced behavioral impairment.

NBP inhibits LPS-induced dopaminergic neuronal loss. TH-positive cells are an important pathological indicator for assessing whether a drug exhibits protective effects on dopaminergic neurons (15). Immunoblotting was also used to analyze the number of TH-positive cells in the SNpc (Fig. 2). In the LPS-treatment group, the number of TH-positive cells in the SNpc $(122.33 \pm 30.29)$ was significantly reduced compared with the control group $(242.33 \pm 19.14 ; \mathrm{P}<0.01)$. Compared with the LPS-treatment group, the number of TH-positive cells in the NBP treatment group (191.67 \pm 23.12$)$ were significantly increased $(\mathrm{P}<0.01)$, indicating that NBP could inhibit the loss of $\mathrm{TH}$.

NBP inhibits LPS-induced inflammatory signaling pathways involving JNK and $p 38$. A previous study demonstrated that the MAPK signaling pathway serves an important role in the pathogenesis of PD (4). To evaluate whether the MAPK 

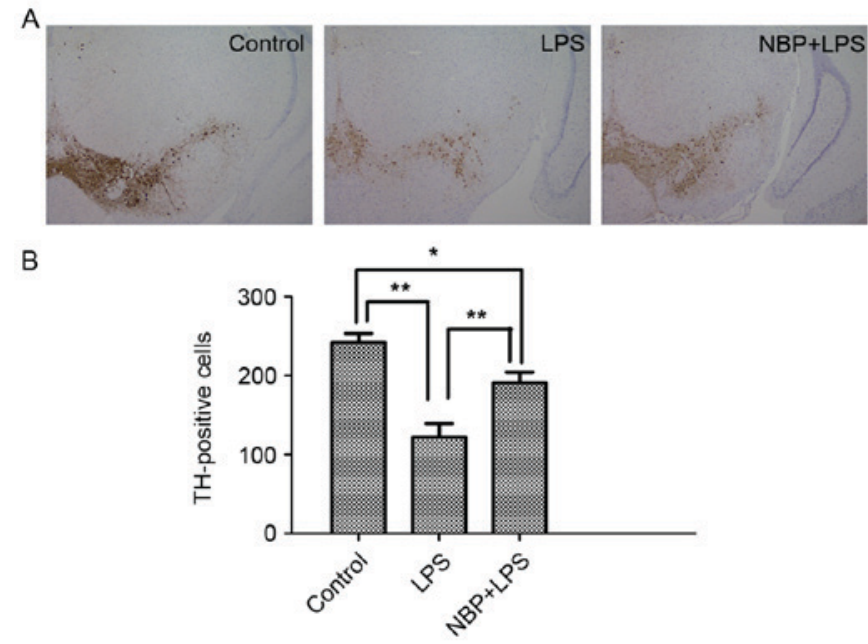

Figure 2. NBP inhibits LPS-induced dopaminergic neuronal loss. TH was detected by immunohistochemical staining in the SNpc of Parkinson's disease model mice. (A) TH-positive cells distribution in the control group, LPS-treatment group and NBP-treatment group. Magnification, x100. (B) Quantification of TH-positive cells in the SNpc for each group. Data are representative of three independent experiments. The values are expressed as mean \pm standard deviation ${ }^{*} \mathrm{P}<0.05,{ }^{* *} \mathrm{P}<0.01$. TH, tyrosine hydroxylase; SNpc, substantia nigra pars compacta; NBP, DL-3-n-butylphthalide; LPS, lipopolysaccharide; PD, Parkinson's disease.

pathway is involved in the protective effect exerted by NBP, the alterations in JNK, p38 and ERK in mice treated with LPS and NBP were investigated.

As demonstrated in Fig. 3, the levels of p-JNK, p-p38 and p-ERK in the control group were $0.23 \pm 0.08,0.28 \pm 0.08$ and $0.99 \pm 0.24$, respectively. The levels of p-JNK, p-p38 and p-ERK in the LPS-treatment group were $0.56 \pm 0.13,0.52 \pm 0.17$, and $1.0 \pm 0.27$, respectively, which indicated phosphorylation of JNK, p38, and ERK, although no significant difference was observed with ERK. The levels of p-JNK, p-p38 and p-ERK in the NBP-treatment group were $0.31 \pm 0.09,0.34 \pm 0.11$, and $0.85 \pm 0.21$, respectively. Compared with the LPS-treatment group, treatment with NBP significantly inhibited phosphorylation of JNK and p38 $(\mathrm{P}<0.05)$, but had little effect on ERK. Compared with the control group, treatment with NBP had no significant effect on the phosphorylation of JNK and p38 ( $>>0.05)$.

Therefore, these results indicated that inhibition of the JNK and p38 pathway is involved in the protective effect of NBP against LPS-induced neurotoxicity.

\section{Discussion}

PD is a common age-associated neurodegenerative disorder, characterized by dopaminergic neuron loss in the SNpc. In the later stages of human PD, there are clear signs of microglial activation and inflammation that may contribute to the progression of the disease $(16,17)$.

The LPS model has been widely used in studies of the biochemical, neurochemical and inflammatory markers, and functional behaviors (18). It has previously been reported that administration of LPS induced symptoms of PD, dopaminergic neuron death and microglial activation $(19,20)$.

NBP has been reported to possess a range of pharmacological effects. It has been demonstrated that NBP protects
A
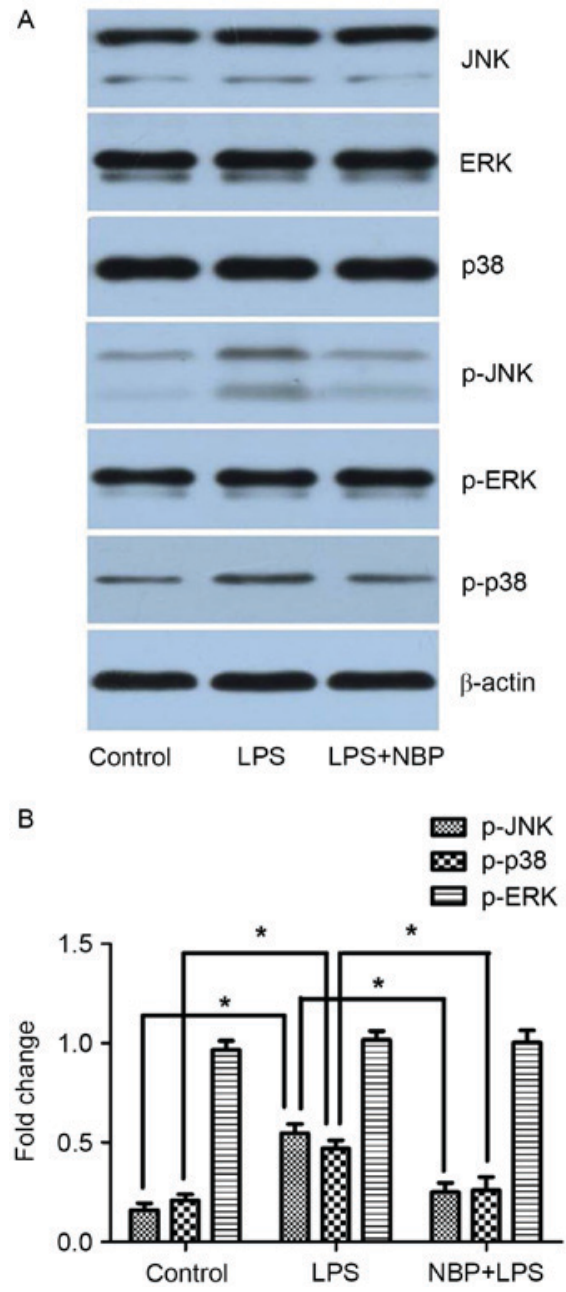

Figure 3.NBP inhibits LPS-induced inflammatory signaling pathways involving JNK and p38. (A) Levels of p-JNK, p38 p-p38 and p-ERK were determined by western blotting. $\beta$-actin was used as an internal control. (B) Band intensities of p-JNK, p-p38 and p-ERK were quantified using densitometry. The values are expressed as mean \pm standard deviation. ${ }^{*} \mathrm{P}<0.05$. Data are representative of three independent experiments. p-, phosphorylated; JNK, c-Jun N-terminal kinase; p38, mitogen-activated protein kinase 14; ERK, extracellular signal-regulated kinase; NBP, DL-3-n-butylphthalide; LPS, lipopolysaccharide.

the brain from ischemia, inhibits platelet aggregation, reduces the area of cerebral infarcts and decreases neuronal apoptosis and oxidative brain damage (10,21-26). Additionally, NBP has also been demonstrated to serve a potential therapeutic role in Alzheimer's and can decrease the cytotoxicity of MPTP in a cellular model of PD (27).

Therefore, in the present study, a purely inflammationdriven (LPS-induced) mouse model of PD was employed. It was also examined whether NBP can suppress inflammatory nerve damage induced by LPS in a mouse model and further investigated the possible mechanisms.

Behavioral tests, including the open-field test and the rotarod test, can be used to observe movement disorders in the mouse. $\mathrm{TH}$ is the restricting enzyme in dopamine synthesis; immunohistochemical analysis of TH allows assessment of the number and function of dopaminergic neurons in the SN (28). Behavioral tests are crucial to evaluate whether a drug exhibits protective effects on dopaminergic neurons, while the number of TH-positive cells is an important pathological indicator. In the present study, the mice treated with LPS exhibited the basic characteristics of PD. 
The mice exhibited movement disorder. The retention times of mice treated with LPS was markedly reduced and the numbers of line crossing, rearing, and grooming actions of mice treated with LPS were decreased. These mice demonstrated a decrease in TH-positive cells. However, the group that received NBP treatment demonstrated an improvement in behavior and increase in TH-positive cells. These results suggested that NBP markedly improved behavior performance and pathological indicator test results in the LPS-induced mouse model of PD, but the exact underlying mechanism remains unclear.

The MAPK superfamily comprises 3 major branches, including JNK, p38 and ERK (29). These subfamilies serve key roles in a variety of cellular responses, including immunity, apoptosis, autophagy, cell survival, cell growth and cell division (29, 30-32).

JNK is markedly activated in models of PD induced by LPS, 6-OHDA and MPTP $(33,34)$. A previous study demonstrated that activation of the JNK pathway is associated with neural cell apoptosis in models of PD (34). The inhibitor of JNK, SR-3306, can reduce the loss of neurons in a rat model of PD, suggesting that JNK inhibitors possess a protective effect on neurons in PD (35). p38 is also activated in neurotoxic PD models (36). Furthermore, inhibitors of $\mathrm{p} 38$ are effective in the treatment of PD (4). ERK is involved in regulating cell proliferation, development and apoptosis. In a cell model of PD, chronic treatment with MPTP can decrease mitochondrial oxidative phosphorylation in dopaminergic neural cells, and inhibiting ERK activation by adding the inhibitor U0126 reversed the mitochondrial morphological alterations and the loss of mitochondrial proteins observed in cells chronically stressed with MPTP (37). A study has also revealed that the ERK signaling pathway may be an important target for the treatment of PD (38).

In the present study, the expression of p-JNK, p-p38 and p-ERK in mice treated with LPS and NBP was observed. The results of the present study revealed that mice treated with NBP demonstrated inhibited LPS-induced phosphorylation of JNK and p38, but had no effect on ERK. These results indicated that neuroprotection mediated by NBP involves the JNK and $\mathrm{p} 38$ pathways.

In conclusion, the present study demonstrated that NBP may serve a valuable neuroprotective role, due to its ability to regulate MAPK pathways in models of PD. These results on the effect of NBP may lay the basis for the development of novel clinical drugs for treating PD.

\section{Acknowledgements}

The present study was supported by the Natural Science Foundation of Anhui Province in 2014 (grant no. KJ2014A163), the National Natural Science Fund of China in 2017 (grant no. 81641050), and the Postgraduate Research and Innovation Project of Bengbu Medical College in 2015 (grant no. Byycxz1506). The authors would like to thank Editage (Cactus Communications, Mumbai, India) for English language editing.

\section{References}

1. Pezzoli G and Zini M: Levodopa in Parkinson's disease: From the past to the future. Expert Opin Pharmacother 11: 627-635, 2010 .
2. Richardson JR and Hossain MM: Microglial ion channels as potential targets for neuroprotection in Parkinson's disease. Neural Plast 2013: 587418, 2013.

3. AlDakheel A, Kalia LV and Lang AE: Pathogenesis-targeted, disease-modifying therapies in Parkinson disease. Neurotherapeutics 11: 6-23, 2014.

4. Wang G, Pan J and Chen SD: Kinases and kinase signaling pathways: Potential therapeutic targets in Parkinson's disease. ProgNeurobiol 98: 207-221, 2012.

5. Whitton PS: Inflammation as a causative factor in the aetiology of Parkinson's disease. Br J Pharmaco 150: 963-976, 2007.

6. Dutta G, Zhang P and Liu B: The lipopolysaccharide Parkinson's disease animal model: Mechanistic studies and drug discovery. Fundam Clin Pharmacol 22: 453-464, 2008.

7. Hines DJ, Choi HB, Hines RM, Phillips AG and MacVicar BA: Prevention of LPS-induced microglia activation, cytokine production and sickness behavior with TLR4 receptor interfering peptides. PLoS One 8: e60388, 2013.

8. Pereira MD, Ksiazek K and Menezes R: Oxidative stress in neurodegenerative diseases and ageing. Oxid Med Cell Longev 2012: 796360, 2012.

9. Imai Y, Venderova K, Park DS, Cai H and Schmidt E: Animal models of Parkinson's disease. Parkinsons Dis 2011: 364328, 2011.

10. Chang Q and Wang XL: Effects of chiral 3-n-butylphthalide on apoptosis induced by transient focal cerebral ischemia in rats. Acta Pharmacol Sin 24: 796-804, 2003.

11. Dong GX and Feng YP: Effects of NBP on ATPase and anti-oxidant enzymes activities and lipid peroxidation in transient focal cerebral ischemic rats. Zhongguo Yi Xue Ke Xue Yuan Xue Bao 24: 93-97, 2002.

12. Li L, Zhang B, Tao Y, Wang Y, Wei H, Zhao J, Huang R and Pei Z: DL-3-n-butylphthalide protects endothelial cells against oxidative/nitrosative stress, mitochondrial damage and subsequent cell death after oxygen glucose deprivation in vitro. Brain Res 1290: 91-101, 2009.

13. Huang JZ, Chen YZ, Su M, Zheng HF, Yang YP, Chen J and Liu CF: DL-3-n-butylphthalide prevents oxidative damage and reduces mitochondrial dysfunction in an MPP(+)-induced cellular model of Parkinson's disease. Neurosci Lett 475: 89-94, 2010.

14. Tian Y, Zhang Y, Zhang R, Qiao S and Fan J: Resolvin D2 recovers neural injury by suppressing inflammatory mediators expression in lipopolysaccharide-induced Parkinson's disease rat model. Biochem Biophys Res Commun 460: 799-805, 2015.

15. Yang W, Chen YH, Liu H and Qu HD: Neuroprotective effects of piperine on the 1-methyl-4-phenyl-1,2,3,6-tetrahydropyridine-induced Parkinson's disease mouse model. Int J Mol Med 36: 1369-1376, 2015.

16. Cebrián C, Loike JD and Sulzer D: Neuroinflammation in Parkinson's disease animal models: A cell stress response or a step in neurodegeneration? Curr Top Behav Neurosci 22: 237-270, 2015.

17. Kannarkat GT, Boss JM and Tansey MG: The role of innate and adaptive immunity in Parkinson's disease. J Parkinsons Dis 3: 493-514, 2013.

18. Tai W, Ye X, Bao X, Zhao B, Wang X and Zhang D: Inhibition of Src tyrosine kinase activity by squamosamide derivative FLZ attenuates neuroinflammation in both in vivo and in vitro Parkinson's disease models. Neuropharmacology 75: 201-212, 2013.

19. Zhou HF, Liu XY, Niu DB, Li FQ, He QH and Wang XM: Triptolide protects dopaminergic neurons from inflammation-mediated damage induced by lipopolysaccharide intranigral injection. Neurobiol Dis 18: 441-449, 2005.

20. Liu B and Hong JS: Role of microglia in inflammation-mediated neurodegenerative diseases: Mechanisms and strategies for therapeutic intervention. J Pharmacol Exp Ther 304: 1-7, 2003.

21. Peng Y, Zeng X, Feng Y and Wang X: Antiplatelet and antithrombotic activity of L-3-n-butylphthalide in rats. J Cardiovasc Pharmacol 43: 876-881, 2004.

22. Yan $\mathrm{CH}$, Feng YP and Zhang JT: Effects of dl-3-n-butylphthalide on regional cerebral blood flow in right middle cerebral artery occlusion rats. Zhongguo Yao Li Xue Bao 19: 117-120, 1998.

23. Liu CL, Liao SJ, Zeng JS, Lin JW, Li CX, Xie LC, Shi XG and Huang RX: DL-3n-butylphthalide prevents stroke via improvement of cerebral microvessels in RHRSP. J Neurol Sci 260: 106-113, 2007.

24. Zhang L, Yu WH, Wang YX, Wang C, Zhao F, Qi W, Chan WM, Huang Y, Wai MS, Dong J and Yew DT: DL-3-n-butylphthalide, an anti-oxidant agent, prevents neurological deficits and cerebral injury following stroke per functional analysis, magnetic resonance imaging and histological assessment. Curr Neurovasc Res 9: 167-175, 2012. 
25. Li J, Li Y, Ogle M, Zhou X, Song M, Yu SP and Wei L: DL-3-n-butylphthalide prevents neuronal cell death after focal cerebral ischemia in mice via the JNK pathway. Brain Res 1359 216-226, 2010.

26. Yan CH, Feng YP and Zhang JT: Effects of dl-3-n-butylphthalide on regional cerebral blood flow in right middle cerebral artery occlusion rats. Zhongguo Yao Li XueBao 19: 117-120, 1998.

27. Peng Y, Xing C, Lemere CA, Chen G, Wang L, Feng Y and Wang X: L-3-n-butylphthalide ameliorates beta-amyloid-induced neuronal toxicity in cultured neuronal cells. Neurosci Lett 434: 224-229, 2008

28. Wang XH, Lu G, Hu X, Tsang KS, Kwong WH, Wu FX, Meng HW, Jiang S, Liu SW, Ng HK and Poon WS: Quantitative assessment of gait and neurochemical correlation in a classical murine model of Parkinson's disease. BMC Neurosci 13: 142, 2012.

29. Kim EK and Choi EJ: Pathological roles of MAPK signaling pathways in human diseases. Biochim Biophys Acta 1802: 396-405, 2010.

30. Corrêa SA and Eales KL: The role of p38 MAPK and its substrates in neuronal plasticity and neurodegenerative disease. J Signal Transduct 2012: 649079, 2012.

31. Cuadrado A and Nebreda AR: Mechanisms and functions of p38 MAPK signalling. Biochem J 429: 403-417, 2010.

32. Cargnello M and Roux PP: Activation and function of the MAPKs and their substrates, the MAPK-activated protein kinases. Microbiol Mol Biol Rev 75: 50-83, 2011.
33. Kaul T, Credle J, Haggerty T, Oaks AW, Masliah E and Sidhu A: Region-specific tauopathy and synucleinopathy in brain of the alpha-synuclein overexpressing mouse model of Parkinson's disease. BMC Neurosci 12: 79, 2011.

34. Huang Q, Du X, He X, Yu Q, Hu K, Breitwieser W, Shen Q, Ma S and Li M: JNK-mediated activation of ATF2 contributes to dopaminergic neurodegeneration in the MPTP mouse model of Parkinson's disease. Exp Neurol 277: 296-304, 2016.

35. Crocker CE, Khan S, Cameron MD, Robertson HA, Robertson GS and Lograsso P: JNK inhibition protects dopamine neurons and provides behavioral improvement in a rat 6-hydroxydopamine model of Parkinson's disease. ACS Chem Neurosci 2: 207-212, 2011.

36. Hwang CJ, Lee HP, Choi DY, Jeong HS, Kim TH, Lee TH, Kim YM, Moon DB, Park SS, Kim SY, et al: Inhibitory effect of thiacremonone on MPTP-induced dopaminergic neurodegeneration through inhibition of p38 activation. Oncotarget 7: 46943-46958, 2016.

37. Zhu JH, Gusdon AM, Cimen H, Van Houten B, Koc E and Chu CT: Impaired mitochondrial biogenesis contributes to depletion of functional mitochondria in chronic $\mathrm{MPP}^{+}$toxicity: Dual roles for ERK1/2. Cell Death Dis 3: e312, 2012.

38. Amiri E, Ghasemi R and Moosavi M: Agmatine protects against 6-OHDA-induced apoptosis, and ERK and Akt/GSK disruption in SH-SY5Y cells. Cell Mol Neurobiol 36: 829-838, 2016. 